hep-th/9507018, RU-95-46

\title{
Chiral Duals of Non-Chiral SUSY Gauge Theories
}

\author{
P. Pouliot \\ Department of Physics and Astronomy \\ Rutgers University \\ Piscataway, NJ 08855-0849, USA
}

\begin{abstract}
We study $N=1$ SUSY gauge theories in four dimensions with gauge group $\operatorname{Spin}(7)$ and $N_{f}$ flavors of quarks in the spinorial representation. We find that in the range $6<$ $N_{f}<15$, this theory has a long distance description in terms of an $S U\left(N_{f}-4\right)$ gauge theory with a symmetric tensor and $N_{f}$ antifundamentals. As a spin-off, we obtain by deforming along a flat direction a dual description of the theories based on the exceptional gauge group $G_{2}$ with $N_{f}$ fundamental flavors of quarks.
\end{abstract}

$6 / 95$ 
Over the past two years, it has become clear that supersymmetry renders possible the precise study of dynamical phenomena in quantum field theory. For recent reviews and lists of references, see [1,2]; for reviews to earlier work, see [3-6]. The outstanding idea in understanding $N=1$ SUSY theories is Duality [7]: a theory which is in a nonAbelian Coulomb phase at large distances may have an equivalent description in terms of a different gauge theory, with different gauge group and matter content. A complicated, strongly coupled theory might have a weakly coupled dual, and that allows the theory to be solved. This duality is a generalization of the Montonen-Olive duality [8,9] of extended supersymmetry.

In attempting to better understand the nature and generality of this $N=1$ nonAbelian duality, a number of examples have been found. One class of examples consists of $S U(N), S O(N)$ and $S p(N)$ gauge theories with matter in the fundamental representations originally found in [7] and further studied in [7, 10,11]. In a second class of examples, one considers a theory under some perturbation of the superpotential [12]. Its effect is to constrain the R-symmetry and to reduce the number of independent chiral operators. The number of examples of this type found so far is impressive [12 18], giving support to the idea that duality is the norm rather than a curiosity, at least for supersymmetric theories [2].

In this letter, we study new examples of duality, without requiring a perturbation by a superpotential. We encounter the situation that vector-like models have chiral duals. In a chiral supersymmetric gauge theory, the chiral superfields (seen as a large reducible representation) do not fall in a real representation of the gauge group. Examples of duality in chiral models have been found in [15, 18]. There, the chiral theories had chiral duals. Nevertheless, there is nothing wrong a priori in a non-chiral theory having a chiral dual. As in [7], we are unable to prove rigorously that the theories we present are dual. However, we give a number of arguments that strongly support it to be the case. The presentation of our results follows closely that of Seiberg's seminal paper [7].

The theory that we study is based on the gauge group $\operatorname{Spin}(7)$, with $N_{f}$ fundamental flavors of quarks, $Q_{f}, f=1, \ldots, N_{f}$. This group is 21 dimensional and the adjoint representation has Casimir index 10; its spinorial representation is real and 8 dimensional and its Casimir index is 2. We refer to this theory as the 'electric' theory. The continuous global symmetry is $S U\left(N_{f}\right) \times U(1)_{R}$, where $U(1)_{R}$ is an R-symmetry. The quantum numbers of the superfields are listed below:

$$
\begin{array}{cccc} 
& \operatorname{Spin}(7) & S U\left(N_{f}\right) & U(1)_{R} \\
Q & 8 & N_{f} & 1-\frac{5}{N_{f}} .
\end{array}
$$


The behavior of this theory for $N_{f} \leq 6$ is standard [3, 19,20], so we will only briefly state the results. The independent gauge invariant operators built out of $Q_{f}$ are a symmetric tensor $M=Q^{2}$ (mesons), and a totally antisymmetric 4-index tensor $B=Q^{4}$ (baryons, for $\left.N_{f} \geq 4\right)$. Classically, there are exactly flat directions of the potential, labeled by the expectation value of $Q_{f}$, and along which the theory follows a

$$
\operatorname{Spin}(7) \rightarrow G_{2} \rightarrow S U(3) \rightarrow S U(2) \rightarrow 1
$$

pattern of symmetry breaking. Using holomorphy and the symmetries, we find that for $N_{f} \leq 3$, a superpotential is generated dynamically:

$$
W_{d y n}=\left(\frac{\Lambda^{15-N_{f}}}{\operatorname{det} M}\right)^{1 /\left(5-N_{f}\right)},
$$

where $\Lambda$ is the dynamical scale of the theory. For $N_{f}=4$ however, the superpotential is determined by holomorphy and the symmetries only up to some function of $\operatorname{det} M / B^{2}$. Observing that when $\operatorname{det} M-B^{2}=0$, an $S U(2)$ subgroup of $\operatorname{Spin}(7)$ remains unbroken classically, it follows by the techniques of [21] that the superpotential has a pole and thus takes the form:

$$
\frac{\Lambda^{11}}{\operatorname{det} M-B^{2}}
$$

(in this expression and elsewhere, for the sake of clarity, we do not write explicitly the numerical coefficients (e.g. permutation symmetry factors) but it is a straightforward matter to determine them). This superpotential is generated by instantons while (3) is generated by gaugino condensation (for $N_{f} \leq 3$ ). These theories do not have a ground state. For $N_{f} \geq 5$, there is no dynamically generated superpotential. For $N_{f}=5$, the fields are classically constrained by the equation $\operatorname{det} M-M_{i j} B^{i} B^{j}=0$, where $B^{i}=\epsilon^{i j k l m} B_{j k l m}$; however, this constraint is modified by instanton effects to $\operatorname{det} M-M_{i j} B^{i} B^{j}=\Lambda^{10}$. For $N_{f} \geq 6$, the quantum moduli space of vacua is the same as the classical one. This can be seen by turning on a mass term $\operatorname{tr} m M$ and adding it to the expression (3). The equations of motion imply $\left\langle M_{i j}\right\rangle=\left(\Lambda^{15-N_{f}} \operatorname{det} m\right)^{1 / 5}\left(m^{-1}\right)_{i j}$ (and $\langle B\rangle=0$ ), so that by taking various limits $m \rightarrow 0$, all classical values of $M$ with $B=0$ can be obtained. For $N_{f}=6$, the expectation values of $M$ and $B$ are constrained by

$$
\operatorname{det} M\left(M^{-1}\right)^{i j}-M_{k l} B^{i k} B^{j l}=0 \quad \text { and } \quad M_{i k} M_{j l} B^{k l}+\epsilon_{i j k l m n} B^{k l} B^{m n}=0,
$$

where $B^{i j}=\epsilon^{i j k l m n} B_{k l m n}$. It can be checked that these constraints properly reduce along the $S U(3)$ flat direction to the constraints on the mesons and baryons of $S U(3)$ with 
4 fundamental flavors [20]. Quantum mechanically however, the theory confines and is described by the unconstrained fields $M$ and $B$ with a superpotential proportional to

$$
\operatorname{det} M-M_{i k} M_{j l} B^{i j} B^{k l}-\operatorname{Pf} B,
$$

whose equations of motion reproduce the constraints (5). This effective theory of mesons and baryons satisfies the 't Hooft anomaly matching conditions at the origin $M=B=0$ where the global symmetry is unbroken.

For $7 \leq N_{f} \leq 14$, the theory at the origin of the moduli space is in an interacting non-Abelian Coulomb phase: the degrees of freedom are the electric quarks and gluons in the infrared. This is clear since for $N_{f} \geq 7$, there is a flat direction along which the group is broken to $S U(3)$ with $5 \leq N_{f}-2$ flavors remaining and these theories are known [20,8] to be in a non-Abelian Coulomb phase. The main claim of this paper is that in the range $7 \leq N_{f} \leq 14$, the electric theory described above has an equivalent description in the extreme infrared in terms of a different, 'dual' gauge theory. We refer to this theory as magnetic and without further ado, state its content. It is an $S U\left(N_{f}-4\right)$ gauge theory with a symmetric tensor $s$, antifundamental quarks $q^{f}, f=1, \ldots, N_{f}$ and mesons $M$, whose transformation properties under the gauge and global symmetries are:

$$
\begin{array}{cccc} 
& S U\left(N_{f}-4\right) & S U\left(N_{f}\right) & U(1)_{R} \\
q & \overline{N_{f}-4} & \overline{N_{f}} & \frac{5}{N_{f}}-\frac{1}{N_{f}-4} \\
s & \frac{\left(N_{f}-4\right)\left(N_{f}-3\right)}{2} & 1 & \frac{2}{N_{f}-4} \\
M & 1 & \frac{N_{f}\left(N_{f}+1\right)}{2} & 2-\frac{10}{N_{f}} .
\end{array}
$$

These fields have interactions dictated by the superpotential

$$
\frac{1}{\mu^{2}} M_{f g} q^{f a} q^{g b} s_{a b}+\frac{1}{\mu^{N_{f}-7}} \operatorname{det} s
$$

where $\mu$ is a dimensionful parameter that is required to give the superpotential a dimension of 3 , since we take the dimension of $M$ to be the one at the ultraviolet fixed point of the electric theory [10]. From this point on, we will set $\mu=1$. Using the techniques of [19], it follows that the unperturbed magnetic superpotential does not receive quantum corrections. As in [7], we are unable to prove that this magnetic theory is dual to the $\operatorname{Spin}(7)$ theory with $N_{f}$ flavors; however, we provide below a number of consistency checks, the sum of which we consider to be strong evidence for duality. 
The $U(1)_{R}$ symmetry so defined is anomaly free. Moreover the 't Hooft anomaly matching conditions are satisfied. They are, in both the electric and magnetic theories: $S U\left(N_{f}\right)^{3}: 8 ; S U\left(N_{f}\right)^{2} U(1)_{R}: \frac{-40}{N_{f}} ; U(1)_{R}:-19 ; U(1)_{R}^{3}: 21-\frac{1000}{N_{f}^{2}}$.

Although one cannot talk about the particle spectrum of such interacting conformal field theories, there should be a one-to-one correspondence that preserves the symmetries between the gauge invariant operators of the electric and magnetic theories. $Q^{2}$ is naturally mapped to $M$ and $B=Q^{4}$ is mapped to $b=q^{N_{f}-4}$. Under this mapping, the symmetries are preserved. The gauge invariant operators $q^{2} s$ and $\operatorname{det} s$ are redundant and vanish identically by the equations of motion.

For $N_{f} \geq 15$, the theory is not asymptotically free, therefore it is a free theory of gluons and quarks in the infrared. The magnetic description should not be valid there. To see this, note that when $N_{f} \geq 15$, the gauge invariant operator qqs has R-charge less than $2 / 3$. By unitarity, it must be a free field if the magnetic theory is in a non-Abelian Coulomb phase. But there is certainly no field with such symmetry properties in the free electric theory, which confirms that the magnetic theory is very strongly coupled for $N_{f} \geq 15$. For $N_{f}=7$, the R-charge of the meson $M$ is $4 / 7<2 / 3$, and thus must be a free field so that the theory be unitary; however the whole theory is not free. There is no value of $N_{f}$ for which the theory is in a free magnetic phase. One might hope that this fact, which makes the analysis more difficult, is not a generic feature of chiral theories. Later we will give an example with a free magnetic description.

We now study the mass perturbations of the $\operatorname{Spin}(7)$ theory. Consider giving a mass $m M_{N_{f}, N_{f}}$ to the last flavor of the $\operatorname{Spin}(7)$ theory with $N_{f}$ flavors, $14 \geq N_{f} \geq 8$. In the infrared, the theory flows to a $\operatorname{Spin}(7)$ theory with $N_{f}-1$ flavors. The effect of this perturbation on the magnetic $S U\left(N_{f}-4\right)$ theory with $N_{f}$ flavors can be found by studying the equations of motion following from the superpotential $m M_{N_{f}, N_{f}}+M q^{2} s+s^{N_{f}-4}$. The equation of motion for $M_{N_{f}, N_{f}}$ is $\left\langle q^{N_{f}} q^{N_{f}} s\right\rangle=-m$. Thus $q^{N_{f}}$ and $s$ acquire expectation values. By a gauge transformation, we can take $\left\langle q^{N_{f}}\right\rangle=(0, \ldots, 0, q)$, with $\left\langle s_{N_{f}-4, N_{f}-4}\right\rangle \neq$ 0 and all other VEVs of $M, s$ and $q$ vanishing. It is clear that all the equations of motion are then satisfied. Thus the magnetic group is higgsed to $S U\left(N_{f}-5\right)$. Along the way, $2 N_{f}-9$ fields have been eaten by the super-Higgs mechanism. They are the $2 N_{f}-8$ fields $s_{N_{f}-4, i}$ and $q^{N_{f}, i}, i=1, \ldots N_{f}-4$, from which one field is subtracted because of the constraint $\left\langle q^{N_{f}} q^{N_{f}} s\right\rangle=-m$. The superpotential becomes $M q^{2} s+s^{N_{f}-5}$, where now the (suppressed) flavor indices run from 1 to $N_{f}-1$ and a scale $\left\langle s_{N_{f}-4, N_{f}-4}\right\rangle$ has been absorbed by a redefinition of $s$ and $q$. This perturbed magnetic theory is just an $S U\left(N_{f}-5\right)$ 
with $N_{f}-1$ flavors and with the appropriate superpotential to be precisely the dual of the $\operatorname{Spin}(7)$ theory with $N_{f}-1$ flavors.

A more careful analysis is required when $N_{f}=7$. Giving a mass $m M_{77}$ to the electric quarks, the magnetic theory is higgsed to $S U(2)$, with 6 doublets (3 flavors) $\hat{q}^{i f}$ and a triplet $\hat{s}^{i j}$ and the mesons $\hat{M}_{f g}(f, g=1, \ldots 6 ; i, j=1,2)$. The dual superpotential becomes $\hat{s}^{2}+\hat{M} \hat{q} \hat{q} \hat{s}$. The field $\hat{s}$ is massive and should be integrated out. The resulting $S U(2)$ theory with 3 flavors is known to confine [20], and to consist of a theory of mesons $\hat{b}^{f g}=\hat{q}^{f i} \hat{q}^{g j} \epsilon_{i j}$. Therefore the dual superpotential should be rewritten in terms of these meson fields and of $\hat{M}_{f g}$. The strong $S U(2)$ dynamics produces a superpotential Pf $\hat{b}$. The result of integrating out $\hat{s}$ is a contribution $\left(\hat{M}_{f g} \hat{q}^{f i} \hat{q}^{g j}\right)\left(\hat{M}_{f^{\prime} g^{\prime}} \hat{q}^{f^{\prime} i} \hat{q}^{g^{\prime} j}\right)=\hat{M}_{f g} \hat{M}_{f^{\prime} g^{\prime}} \hat{b}^{f f^{\prime}} \hat{b}^{g g^{\prime}}$. We also add a new contribution $\operatorname{det} \hat{M}$, which is consistent with the symmetries. We have not identified the dynamical mechanism generating it. After identifying the mesons $\hat{b}$ to the baryons $B$ of the confining $\operatorname{Spin}(7), N_{f}=6$ theory, the superpotential that results is $\operatorname{det} M-M M B B-\operatorname{Pf} B$ which is the correct superpotential (6). Therefore, by integrating out a quark from the $\operatorname{Spin}(7), N_{f}=7$ theory, both the electric and magnetic theories flow to the confining $\operatorname{Spin}(7), N_{f}=6$ theory. And then, by giving masses and integrating out quarks from this $N_{f}=6$ theory, all the results listed above for $N_{f}<6$ are recovered.

We now study a dual pair which follows from our results for $\operatorname{Spin}(7)$. We consider taking as the electric theory a theory with gauge group $S U\left(N_{f}-4\right)=S U\left(N_{c}\right), N_{c} \geq 3$, and with $N_{f}$ fundamentals $Q$ and a conjugate symmetric tensor $S$. Along the lines of [12], we add a superpotential det $S$ to this electric theory. Its field content and symmetries are

$$
\begin{array}{cccc} 
& S U\left(N_{c}\right) & S U\left(N_{c}+4\right) & U(1)_{R} \\
Q & N_{c} & N_{c}+4 & \frac{5}{N_{c}+4}-\frac{1}{N_{c}} \\
S & \frac{N_{c}\left(N_{c}+1\right)}{2} & 1 & \frac{2}{N_{c}} .
\end{array}
$$

It turns out that this theory is much easier to analyze than when $\operatorname{Spin}(7)$ was the electric theory. The magnetic theory is trivially determined to be:

$$
\begin{array}{cccc} 
& \operatorname{Spin}(7) & S U\left(N_{c}+4\right) & U(1)_{R} \\
q & 8 & \overline{N_{c}+4} & 1-\frac{5}{N_{c}+4} \\
M & 1 & \frac{\left(N_{c}+4\right)\left(N_{c}+5\right)}{2} & \frac{10}{N_{c}+4}
\end{array}
$$

with the simple superpotential $M q q$. The R-symmetry is anomaly free and the 't Hooft anomaly matching conditions are satisfied. The gauge invariant chiral operators are in 
one-to-one correspondence: $M=Q Q S \rightarrow M, Q^{N_{c}} \rightarrow q^{4} ; q q$ and $\operatorname{det} S$ are redundant. In both theories, there is no quantum corrections to the unperturbed superpotentials. When $N_{c} \geq 11$, we find that this electric theory with a symmetric tensor and fundamentals is at very strong coupling, but that it has a magnetic description which is free.

To analyze the flat directions of the electric theory, it is convenient to use the most general solution of the $D$-terms for $S$ and $Q$ found in 4 :

$$
Q_{i f}=\left(\begin{array}{cccc}
Q_{1} & & & \\
& Q_{2} & & \\
& & \ldots & \\
& & & Q_{N_{c}}
\end{array}\right) \quad S=\operatorname{diag}\left(S_{1}, \ldots, S_{N_{c}}\right)
$$

where $\left|Q_{i}\right|^{2}-\left|S_{i}\right|^{2}=$ constant for $i=1, \ldots, N_{c}$. This is easily seen by first diagonalizing the hermitian matrix $S^{\dagger i k} S_{k j}$.

It is necessary for consistency that the flat directions of the dual theories be precisely the same. From the equation of motion for $S$, we find that $S$, and thus $M=Q Q S$, have rank at most $N_{c}-2$ in the electric theory. It is easy to see that $M$ in the magnetic theory also does not have rank larger than $N_{c}-2$. If its rank were larger than $N_{c}-1$, the dual $\operatorname{Spin}(7)$ theory, after integrating out the massive dual quarks, would have less than 5 dual quarks remaining. This $\operatorname{Spin}(7)$ theory generates the superpotential (3) which removes all the vacua. If its rank were $N_{c}-1$, the $\operatorname{Spin}(7)$ theory with 5 remaining dual quarks would have to obey the constraint $\operatorname{det} N-N b b=\widetilde{\Lambda}^{10}$, with $N=q q$ and $b=q^{4}$; however, $N=0$ by the equation of motion for $M$. If the rank of $M$ is less than $N_{c}-1$, the dual $\operatorname{Spin}(7)$ theory keeps its classical flat directions as explained earlier. This shows that the mesonic flat directions are the same in the electric and magnetic theories. To summarize this example, a chiral theory with a superpotential has a simpler, vector-like dual.

We consider now the deformation of the $\operatorname{Spin}(7)$ electric theory along its $G_{2}$ flat direction. Essential results on the group theory of $G_{2}$ will be obtained with little work. We only need to know that $G_{2}$ is the subgroup of $\operatorname{Spin}(7)$ left unbroken when a spinor gets a VEV, that its fundamental representation is 7 dimensional and its adjoint 14 dimensional. Starting with $\operatorname{Spin}(7)$ with $N_{f}+1$ flavors, $Q_{0}$ and $Q_{i}, i=1, \ldots, N_{f}$, consider giving an expectation value to the meson $\left\langle M_{00}\right\rangle \neq 0$, while other $M$ have vanishing expectation value. The electric theory is $G_{2}$ with $N_{f}$ flavors of quarks $Q$, in the 7 dimensional fundamental representation of $G_{2}$ and transforming as

$$
\begin{array}{cccc} 
& G_{2} & S U\left(N_{f}\right) & U(1)_{R} \\
Q & 7 & N_{f} & 1-\frac{4}{N_{f}}
\end{array}
$$


Since the spinor decomposes as $8=1+7$ and the adjoint as $21=14+7$, the Casimir index of the 7 of $G_{2}$ is 2 , while that of the 14 of $G_{2}$ is $10-2=8$. Thus this model is asymptotically free for $N_{f}<12$. We now study the effect of the $\left\langle M_{00}\right\rangle$ perturbation on the dual. From the equation of motion for $q^{i}, M_{i 0}=0$. The superpotential becomes

$$
q^{0} q^{0} s+s^{N_{f}-3}+M q q s
$$

where we have absorbed the scale $\left\langle M_{00}\right\rangle$ in the definition of $q^{0} \equiv q^{N_{f}+1}$. Thus, the dual of $G_{2}$ with $N_{f}$ flavors consists of the fields $q, q^{0}, s$ and $M$ transforming as

$$
\begin{array}{cccc} 
& S U\left(N_{f}-3\right) & S U\left(N_{f}\right) & U(1)_{R} \\
q & \overline{N_{f}-3} & \overline{N_{f}} & \frac{3}{N_{f}}\left(1-\frac{1}{N_{f}-3}\right) \\
q^{0} & \overline{N_{f}-3} & 1 & 1-\frac{1}{N_{f}-3} \\
s & \frac{\left(N_{f}-3\right)\left(N_{f}-2\right)}{2} & 1 & \frac{2}{N_{f}-3} \\
M & 1 & \frac{N_{f}\left(N_{f}+1\right)}{2} & 2-\frac{8}{N_{f}} .
\end{array}
$$

In a similar way to what we discussed for $\operatorname{Spin}(7)$, a number of consistency checks of the duality $G_{2}-S U\left(N_{f}-3\right)$ can be performed. The R-symmetry is anomaly free, the 't Hooft anomaly matching conditions are satisfied, etc. Consider integrating out flavors from the electric theory with $N_{f}$ flavors. When $N_{f} \geq 7$, the effect on the dual is to higgs the gauge group $S U\left(N_{f}-3\right) \rightarrow S U\left(N_{f}-4\right)$, with the dual quark $q^{N_{f}}$ becoming massive. The case $N_{f}=6$ deserves more attention. The dual $S U(2)$ theory that is obtained has six doublets $q^{0}$ and $q^{i}, i=1, \ldots, 5$. Its physics is that of confinement. The correct degrees of freedom in the infrared are the mesons $B^{i}=q^{0} q^{i}$ and $A^{i j}=q^{i} q^{j}$ along with $M_{i j}$. Since by duality this describes the $G_{2}$ theory with 5 flavors, we learn that the independent gauge invariant chiral operators of $G_{2}$ are a 2-index symmetric tensor $M$ of $S U\left(N_{f}\right)$, a 3-index totally antisymmetric tensor $A$ and a 4-index totally antisymmetric tensor $B$. Define an antisymmetric tensor $V$ by $V^{i j}=A^{i j}, i, j=1, \ldots, 5, V^{i 6}=B^{i}, i=1, \ldots, 5$. The $S U(2)$ dynamics gives a contribution to the superpotential of $\operatorname{Pf} V=\epsilon_{i j k l m} B^{i} A^{j k} A^{l m}$. Integrating out $s$, which is massive, produces two more terms $M_{i j} M_{k l} A^{i k} A^{j l}+M_{i j} B^{i} B^{j}$, and we also add $\operatorname{det} M$, allowed by the symmetries, as in the $\operatorname{Spin}(7)$ case with $N_{f}=7$ discussed above. The resulting superpotential

$$
\operatorname{det} M+M_{i j} M_{k l} A^{i k} A^{j l}+M_{i j} B^{i} B^{j}+\epsilon_{i j k l m} B^{i} A^{j k} A^{l m}
$$


describes the confining $G_{2}$ theory with 5 flavors whose equations of motion give the following constraints on the expectation values of $M, A$ and $B$ :

$$
\begin{gathered}
\operatorname{det} M\left(M^{-1}\right)^{i j}+M_{k l} A^{i k} A^{j l}+B^{i} B^{j}=0 \quad \text { and } \\
M_{i k} M_{j l} A^{k l}+\epsilon_{i j k l m} B^{k} A^{l m}=0 \quad M_{i j} B^{j}+\epsilon_{i j k l m} A^{j k} A^{l m}=0 .
\end{gathered}
$$

Adding $m M_{55}$ to (15) to integrate out the fifth flavor, the equation of motion for $M_{55}$ gives a constraint $\operatorname{det} M^{(4)}+M_{i j}^{(4)} A^{(4) i} A^{(4) j}+B^{(4)} B^{(4)}=m$, where the label (4) refers to the subset of fields that belong to the effective theory with 4 flavors. We learn from this constraint that the quantum moduli space is smooth. Keeping $M_{55}$ as a Lagrange multiplier, we obtain the superpotential $W_{(4)}=M_{55}\left(\operatorname{det} M^{(4)}+M^{(4)} A^{(4)} A^{(4)}+B^{(4)} B^{(4)}+m\right)$. Integrating out the fourth quark by adding $m M_{44}$, we obtain a superpotential $W_{(3)}=1 /\left(\operatorname{det} M^{(3)}+\left(A^{(3)}\right)^{2}\right)$. Had we carefully kept track of all the scales, we would have seen that this superpotential has the correct power of the dynamical scale of the electric theory to be generated by instantons. Integrating out the two other flavors lead to superpotentials $W_{(i)}=1 /\left(\operatorname{det} M^{(i)}\right)^{1 /(4-i)}$, $i=1,2$. It is generated by gluino condensation in the $S U(3)$ subgroup of $G_{2}$ for $i=1$ and in $S U(2) \in S U(3)$ for $i=2$. For $i=1,2,3$, there is no vacuum state. Integrating out all the quarks result in gluino condensation in the pure $G_{2}$ gauge theory.

As for future directions, it would be especially interesting, by going up a few more steps, to reach the $S O(10)$ gauge theories with spinors, where the issues of grand unification, duality and dynamical supersymmetry breaking possibly meet.

After the completion of this work, we learned of references [22-24] which also examined some aspects of $G_{2}$ gauge theories.

\section{Acknowledgments}

We would like to thank V. Brazhnikov, K. Intriligator, N. Seiberg and M. Strassler for useful discussions, and especially N. Seiberg for very valuable comments on the manuscript. This work was supported in part by DOE grant \#DE-FG05-90ER40559 and by a Canadian 1967 Science fellowship. 


\section{References}

[1] N. Seiberg, the Power of Holomorphy - Exact Results in 4D SUSY Field Theories, Proc. of PASCOS 94, hep-th/9408013, RU-94-64, IASSNS-HEP-94/57

[2] N. Seiberg, the Power of Duality - Exact Results in 4D SUSY Field Theories, Proc. of PASCOS 95 and Proc. of the Oskar Klein Lectures, hep-th/9506077, RU-95-37, IASSNS-HEP-95/46

[3] I. Affleck, M. Dine and N. Seiberg, Nucl. Phys. B241 (1984) 493

[4] I. Affleck, M. Dine and N. Seiberg, Nucl. Phys. B256 (1985) 557

[5] A.I. Vainshtein, V.I. Zakharov and M.A. Shifman, Sov. Phys. Usp. 28 (1985) 709

[6] D. Amati, K. Konishi, Y. Meurice, G.C. Rossi and G. Veneziano, Phys. Rep. 162 (1988) 169

[7] N. Seiberg, Nucl. Phys. B435 (1995) 129

[8] C. Montonen and D. Olive, Phys. Lett. 72B (1977) 117

[9] N. Seiberg and E. Witten, Nucl. Phys. B426 (1994) 19; Nucl. Phys. B431 (1994) 484

[10] K. Intriligator and N. Seiberg, hep-th/9503179, RU-95-3, IASSNS-HEP-95/5; hepth/9506084, RU-95-40, IASSNS-HEP-95/48

[11] K. Intriligator and P. Pouliot, hep-th/9505006, RU-95-23

[12] D. Kutasov, hep-th/9503086, EFI-95-11

[13] O. Aharony, J. Sonnenschein and S. Yankielowicz, hep-th/9504113, TAUP-2246-95, CERN-TH/95-91

[14] D. Kutasov and A. Schwimmer, hep-th/9505004, EFI-95-20, WIS/4/95

[15] M. Berkooz, hep-th/9505067, RU-95-29

[16] K. Intriligator, hep-th/9505051, RU-95-27

[17] R.G. Leigh and M.J. Strassler, hep-th/9505088, RU-95-30

[18] K. Intriligator, R.G. Leigh and M.J. Strassler, hep-th/9506148, RU-95-38

[19] N. Seiberg, Phys. Lett. 318B (1993) 469

[20] N. Seiberg, Phys. Rev. D49 (1994) 6857

[21] K. Intriligator, R.G. Leigh and N. Seiberg, Phys. Rev. D50 (1994) 1092

[22] E. Witten, unpublished

[23] I. Pesando, hep-th/9506139, NORDITA-95/42 P

[24] S.B. Giddings and J.M. Pierre, hep-th/9506196, UCSBTH-95-14 